\title{
Molecular and crystal assembly inside the carbon nanotube: encapsulation and manufacturing approaches
}

\author{
Sergio Manzetti
}

Received: 30 April 2013/ Accepted: 4 June 2013/Published online: 28 June 2013

(c) Shanghai University and Springer-Verlag Berlin Heidelberg 2013

\begin{abstract}
Encapsulation of different guest-species such as molecules and ions inside carbon nanotubes (CNTs) has been reported in the literatures during the last 15 years and represents an exciting development of nanoengineering of novel materials and composites. The reported nanocomposite materials show the semi-conducting properties with potential applications in nanosensors, nanounits and nanocircuits as well as advanced energy transfer and storage properties, and encompass manufacturing for novel nanowires, nanoelectronic devices with properties designed with optoelectronic, spintronic and nanomagnetic qualities. This review reports on a wide range of encapsulation references with particular focus on single molecules, atomic chains, metal halides and polymers encapsulated inside CNTs. The encapsulation methods and the chemical and physical qualities of these novel materials are crucial for the future manufacturing of novel innovations in nanotechnology, and represent therefore the current state-ofthe-art of encapsulation methods in advanced manufacturing.
\end{abstract}

Keywords Encapsulation - Carbon nanotubes (CNTs) Composites conductive $\cdot$ Molecules

\section{Introduction}

Encapsulation of molecules and crystal assemblies inside carbon nanotubes $(\mathrm{CNT})$ has recently attracted a great deal of

S. Manzetti $(\bowtie)$

Fjordforsk A.S., Institute of Science and Technology,

6896 Fresvik, Norway

e-mail: S.manzetti@fjordforsk.no

\section{S. Manzetti}

Van der Spoel Lab. Computational Systems and Biology, Biomedical Centre, University of Uppsala, P. O. Box 256, 75105 Uppsala, Sweden attention in the field of nanomaterials, nanocomposites, nanowires and selective catalysis [1-4]. The encapsulated composites have been demonstrated to retain their electronic and magnetic properties, which prove useful for spintronics and energy-related applications [4], such as quantum cascade lasers and high-energy technologies [5-7]. Based on their chemistry and electrostatic interactions, the encapsulated species have the possibility of supplying high-energy electrostatic environments for intra-tubular conductance and lowering band gaps by the electronic interaction energy between the intra-face of the nanotubes and the encapsulated assemblies [8, 9]. However, a series of challenges and obstacles are encountered during preparation of such advanced nanocomposites, such as annealing temperatures, effect on encapsulated particle size and stacked and unorganized arrangement of molecules in CNTs. Other challenges are the e-beam irradiation intensity affecting the chemical stability of the reactants during insertion and the effects arising from clustering and grinding of the materials for encapsulation at low, medium and high temperatures. Therefore, this paper presents a critical and methodic overview of different encapsulation techniques for single molecules, molecular chains and crystal assemblies into onedimensional tubular carbon nanostructures such as CNTs and carbon nanofibers (CNF) towards improved nanomaterials and manufacturing approaches.

\section{Single molecules and small particles encapsulated inside CNTs}

The insertion of single molecules in CNTs has been performed by various groups in the last 15 years, and the first experiments showed rows of closed carbon shells, named "peapods" formed inside CNTs after synthesis through 
pulse-laser vaporization [10]. The named peapods which was identified to be fullerene cages was encapsulated inside the CNTs, and organized in vacuum spaces inside the tubular structures, stabilized by van der Waal forces. Other studies in this time-frame reported the encapsulation of small carbon-fragments as co-synthesized products inside CNTs [11], where researchers found that molecular debris from CNT-synthesis entered spontaneously inside the CNTs during CNT-growth [11]. In these various studies, it was found that small CNT fragments entered or deposited inside CNT during synthesis, creating fabricates with different structural and conductive properties deviating from the clean hollow CNTs structures originally expected. In order to actively encapsulate molecules, other groups attempted therefore the insertion of small and larger molecules inside CNTs.

Takenobu et al. [12] published a leading work in 2003 regarding the encapsulation of a selection of organic molecules inside CNTs. These compounds were anthracene, tetracene and pentacene, and fullerene $\mathrm{C}_{60}$ among other tested molecules, which were inserted into the CNT as solids (powder) loaded in sealed glass tubes during prolonged exposures to heat. The encapsulated molecules were purified prior to insertion through sublimation. Heating of the mixture of CNT and the organic compounds was then carried out in order to allow the purified organic compounds to spontaneously enter the CNTs [12] using a tube furnace. The conductive and electronic properties of the obtained composite material were studied revealing charge-transfer interactions occurrence between the intratubular facet of the CNT and the inserted molecules. In the particular case of one of the inserted molecules, tetracyano$p$-quinodimethane, the conductive change on the CNT induced a hole-doping effect, increasing the conductivity of the CNT by Fermi-hole conduction [12]. The chargetransfer studied by Takenobu et al. [12] showed furthermore that the pristine CNTs exhibited $p$-type operation (hole), while CNT filled with tetrathiafulvalene and tetramethyltetraselenafulvalene molecules exhibited $n$-type operation (conduction through free electrons).

Charge-transfer is a crucial part in the fabrication of novel materials with conductive properties, and its relevance with CNTs is of pivotal point for the development of new materials. The study by Takenobu et al. [12] revealed that the charge-transfer interactions between the host CNTs and the guest organic molecules were considerably dependent on the relative ionization energy of the inserted molecules. In addition the electron affinities of the inserted compounds played a substantial role in the electronic properties of the CNT-composite. Indeed, both the relative ionization potentials and the electronic affinities varied significantly by the atomic species contained in these inserted molecules (nitrogen, oxygen or halogen containing molecules), directly affecting the conductivity of the materials. The findings suggested that the chemical properties of the inserted molecules had a direct impact on the conductivity type of the CNT for a potential application in nanoelectronic devices, also including their bond-orders $(\pi$, $\sigma$-bonds), dipole moments, electrostatic properties and localized energies.

Another study reporting the successful insertion of molecules in CNTs was the inclusion of $o$-carborane molecules in CNTs [13]. Morgan et al. [13] used a similar approach as Takenobu et al. [12] by heating $50 \mathrm{~g} o$-carborane with $100 \mathrm{~g}$ CNT in a furnace at $350{ }^{\circ} \mathrm{C}$ under a dynamic atmosphere of argon and oxygen. High-resolution transmission electron microscopy (HRTEM) confirmed the composite structure, giving rise to filling yields ranging between $5 \%$ and $20 \%$, which was subsequently increased when the CNTs were pre-washed with hydrochloric acid. By the method of Ref. [13], the increase of temperature up to $420{ }^{\circ} \mathrm{C}$ did not promote a higher concentration of composite structure with $o$-carborane@CNT, indicating significant limits in temperature modulation when encapsulating materials in CNTs. A wider temperature range for encapsulation was though possible with fullerenes as the encapsulated materials [14], showing higher encapsulationyield with pristine $\mathrm{C}_{60}$ fullerenes over CNTs.

The study by Morgan et al. [13] also showed that sublimation techniques gave a higher yield of composite, in a similar fashion as Takenobu et al. [12] who used sublimation to purify the molecules prior to encapsulation (see Fig. 1). Sublimation is the most appropriate approach in furnace-dependent procedures (different from laser and e-beam irradiation techniques), where the rapid transfer from solid to gaseous phase yields a simultaneous insertion of molecules inside CNTs. Conclusively, sublimation in this methodological context may be regarded as purification + insertion in the same step.

Smith et al. [10] encapsulated larger molecules inside CNTs, reporting a co-synthesis of fullerene $\mathrm{C}_{60}$ inside CNTs during pulsed-laser vaporization technique of a catalystcontaining graphite target. The procedure (see Fig. 2) resulted in a co-synthesis of fullerene particles separated by $0.3 \mathrm{~nm}$ inside the grown CNTs, merged to a contiguous carbon assembly during the application of high-energy electron beams. This growth of fullerenes inside CNTs was then re-investigated by Sloan et al. [17] and also contemporary studied by Zhang et al. [18], who also observed fullerenes in as-prepared CNTs formed by $\mathrm{Ni} / \mathrm{Y}$ catalyzed carbon arc evaporation and defined this as a defect in synthesis. Indeed occurring as a defect, the presence of encapsulated fullerenes showed to alter the conductive properties of CNTs and affect the materials physical qualities (see Fig. 3). However, Smith et al. [14] reported a rapid coalescence of the encapsulated fullerenes when subjected to high 


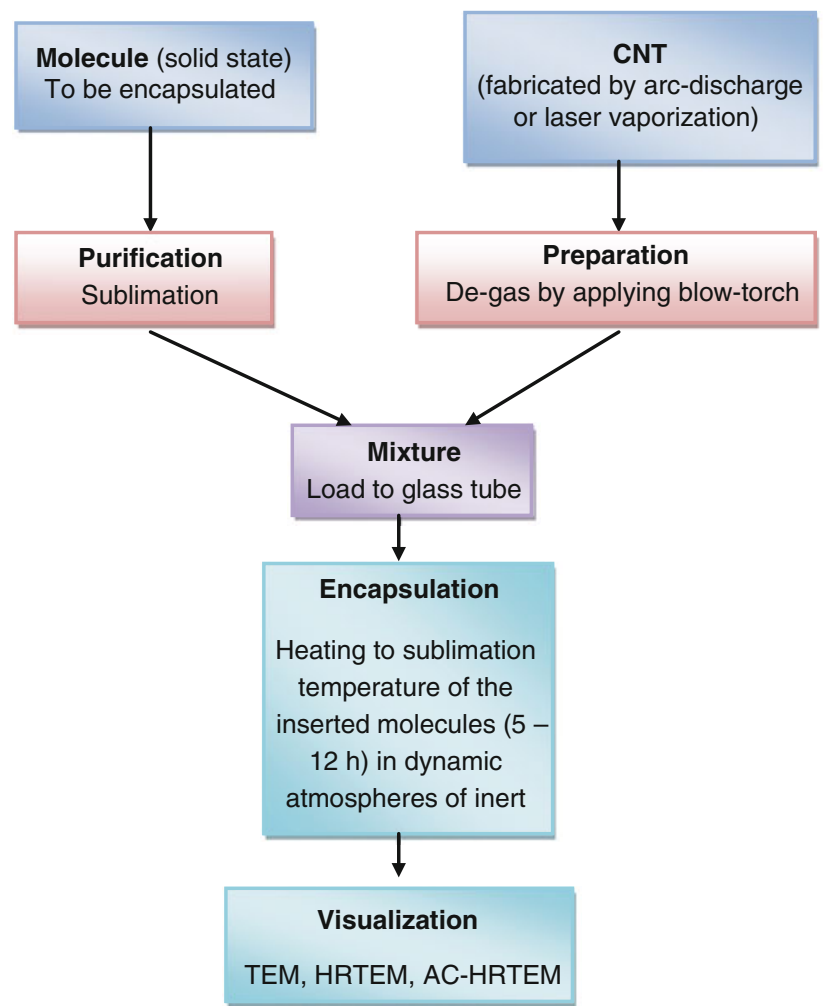

Fig. 1 Main steps describing the protocol for encapsulation of single molecules in CNTs [12]

electron flux in a $300 \mathrm{kV}$ field emission gun transmission electron microscope (FEGTEM) for $5 \mathrm{~min}$. The procedure applied by Smith et al. [14] to generate $\mathrm{C}_{60}$ chains inside the CNT encompassed the use of $100 \mathrm{~mm}$ long $10 \mathrm{~mm}$ diameter graphite rod, which were processed down to a diameter of $6 \mathrm{~mm}$ with a $40 \mathrm{~mm}$ deep $3.5 \mathrm{~mm}$ diameter hole, and drilled along the central axis of the thin region. The cavity was packed with a mixture of 4.2 at $\% \mathrm{Ni}$ and 1.0 at\% $\mathrm{Y}$ mixed intimately with graphite powder (cobalt and bismuth can be used as well [15]). The composite was then arc-evaporated in a Krätschmer-Huffman soot box in a dynamic vacuum with an $8.80 \mathrm{kPa} \mathrm{He}$, a voltage of $25-30 \mathrm{~V}$ and a current of 110-140 A. This procedure gave a series of encapsulated fullerenes at a yield of 5\%-10\% packed in continuous chains with well defined separations. Interestingly, a variety of sizes of fullerenes, both smaller and larger than regular $\mathrm{C}_{60}$, ranging from $\mathrm{C}_{32}$ to $\mathrm{C}_{120}$ were obtained with this procedure. The described coalescing procedure (vide supra) was applied to convert the chains of $\mathrm{C}_{60}$ to a regular CNT, encapsulated within the original CNT (yielding a doubleCNT) and gave at first dimers and then trimers within the CNT capillary, followed by a full conversion into a continuous CNT structure. The resulting complexes of $\mathrm{C}_{60} @ \mathrm{CNT}$ indicate that fullerene molecules are trapped within the CNTs by the filling media independent of the method used either through growth or evaporation, and that these are

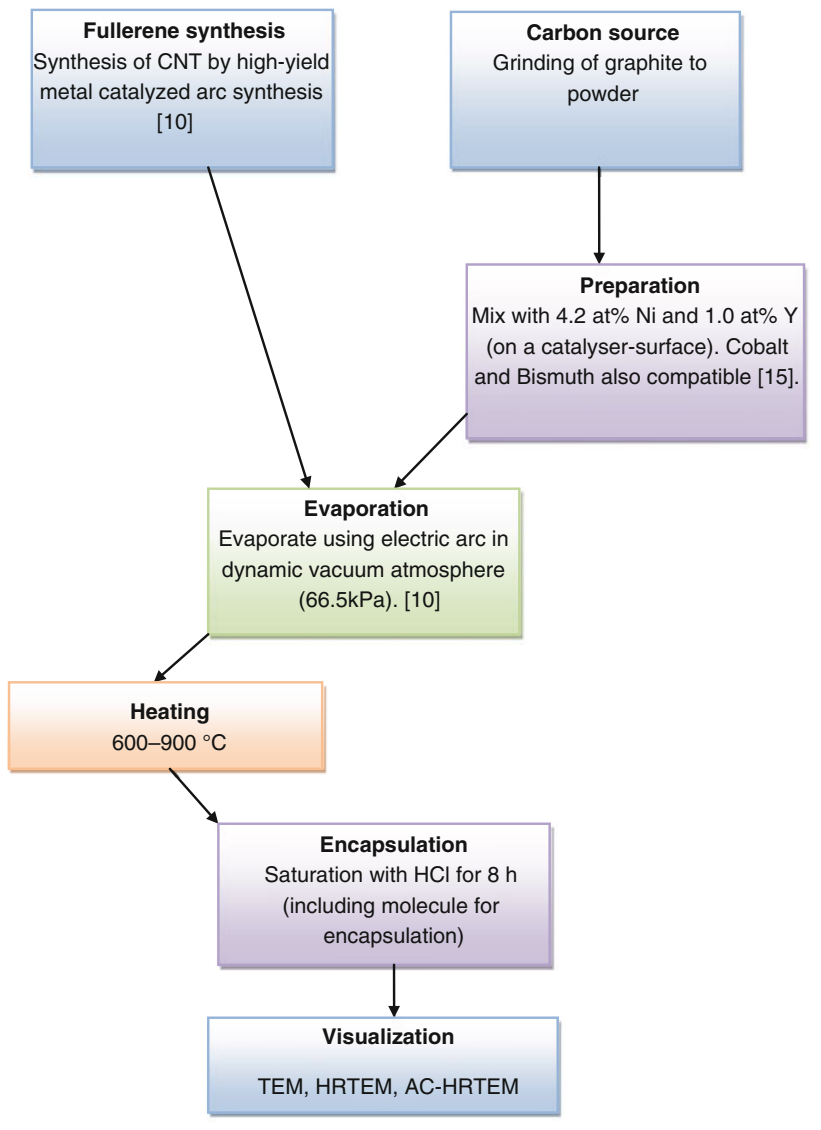

Fig. 2 Main steps describing the protocol for growth of fullerenes inside CNTs [16, 17]

further transformed into different structural ensembles during continuous gradual irradiation.

\section{Encapsulation of linear carbon chains, sheets and oligomers in CNTs}

The first carbon-based linear chains successfully inserted (co-synthesized) in CNTs were short carbon chains, spontaneously formed as deposits from a double-anode arcdischarge process [19]. These deposits were analyzed using electron microscopy, showing a majority of nanostructures to be arranged in a closed fashion, or arranged as threedimensional graphitic sheets and as multi-walled carbon nanotubes (MWCNTs), as also achieved by others later [20]. However Wang et al. [19] observed that the nanowires formed spontaneously inside CNTs organized as needle-like structures. These encapsulated unique structures were composed of a $\mathrm{C}_{n}$-chain within the nanotubes, at a distance of $3.4 \AA$ from the CNT inner wall [19]. The mechanism for growth of carbon "needles" was dependent on the graphite arc-discharge method to produce $\mathrm{C}_{2}$ moieties, which were then linked to active sites of soot 


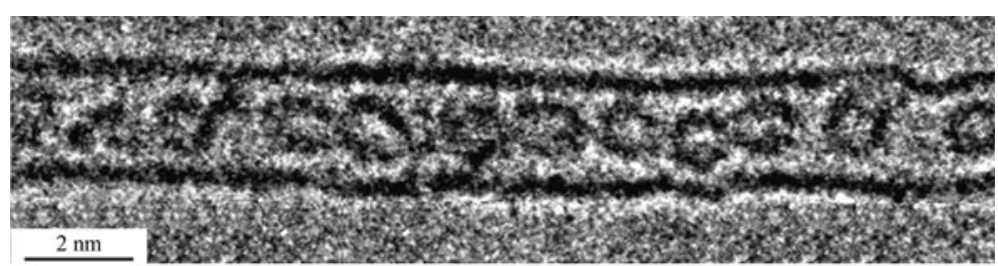

(a)

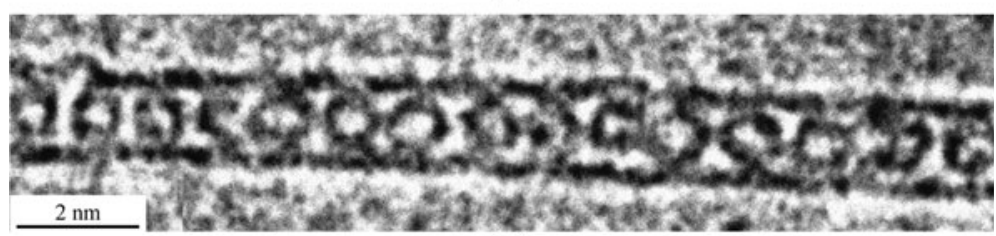

(b)

Fig. 3 Encapsulation of fullerenes inside CNTs (courtesy of Dr. Jeremy Sloan, University of Warwick, Warwick Centre of Analytical Science). Images taken with TEM show uneven-sized fullerenes (a) and even-sized fullerenes (b), using a $300 \mathrm{kV}$ e-beam on the JEOL $3000 \mathrm{~F}$ microscope taken at the Department of Materials at Oxford University

particles. During the assembly into CNTs, a key intermediate, a half- $\mathrm{C}_{60}$ cage was formed, in a similar fashion to the studies by Sloan et al. [17] and Smith et al. [10]. (see previous section), where the center of these half- $\mathrm{C}_{60}$ cages spontaneously attracted carbon atoms from the discharge process [19]. The additional atoms could thus interact with new single carbons, growing the atomic chain from the half- $\mathrm{C}_{60}$ cage. This mechanism assumes by that the newly added atom is stabilized by electrostatic interactions with the half- $\mathrm{C}_{60}$ cage [19]. Resulting a needle-chains, the carbon atoms assembled from the $\mathrm{C}_{60}$ half cage throughout the co-formed CNT, creating a different composite from regular CNTs, with similar properties to MWCNTs [19].

Warner et al. [20] used a different approach from Wang et al. [19] and synthesized encapsulated carbon chains, linear benzene-chains, alkane-chains and polyyne chains (also accomplished by Nishide et al. [21]) inside in CNTs. The generated encapsulated chains were classified as "aggregates" encapsulated within CNTs of diameters of $2-3 \mathrm{~nm}$ at lengths of $1-6 \mathrm{~nm}$. Zhao et al. [22] also described a similar approach to Warner et al. [20], reporting the formation of $20 \mathrm{~nm}$-length carbon nanowires with approximately 100 carbon atoms in a $1 \mathrm{D}$ orientation inside MWCNTs. In addition, Sheng et al. [23] proposed very recently a similar method for the full growth of carbon chains within MWCNTs. Their method employed the synthesis of a 1D sp-hybridized carbon chain (poly-yne chain), followed by growing CNTs using a Raman scattering technique. With their method for composite manufacturing, Sheng et al. [23] observed that carbon structures formed on the outside of the CNT and migrated in the CNT under high temperatures, as also observed in similar experiments with CNT-structures [24]. The thermochemical effect on proper growth of polymers/oligomers in CNTs is thus pivotal, and suggested to be ideal at $1,500{ }^{\circ} \mathrm{C}[23]$ in order to achieve these products of synthesis. Growth of polymers, ribbons and larger molecular assemblies have also been performed recently, with the impressive achievement of Chamberlain et al. [25] who explored the formation of large molecular groups inside CNTs. The growth of molecular systems inside CNT was performed using tetrathiafulvalene, which yielded the formation of helix-twisted sheets encapsulated inside the CNTs, mostly composed of carbon. As in the study by Sheng et al. [23], this group also applied very high temperatures during the treatment, where the thermal treatment under argon or e-beam irradiation in vacuum purged the tetrathiafulvalene (TTF) molecules to decompose and transform into CNTencapsulated sulfur-terminated nanoribbons. Smith et al. [14] found that tubules prepared via pulsed laser vaporization (PLV) contained close-packed 1D chains consisting of $\mathrm{C}_{60}$ and other fullerene-molecules (i.e., $\mathrm{C}_{n}$ ), termed "peapods" or, more formally, $\mathrm{C}_{n} @$ single-walled carbon nanotubes (SWCNTs) [10].

In context with manufacturing conductive materials, Chuvilin et al. [26] engineered a self-assembled chain of carbons atoms, grown inside SWCNTs (see Fig. 4). The arrangement of atoms yielded a nanoribbon structure encapsulated in the CNT, with its ends capped with sulfur atoms, for the special compatibility with molecular contacts. The method of self-assembling the nanoribbons was derived from inserting fullerene-molecules followed by applying either heat $\left(1,000{ }^{\circ} \mathrm{C}\right)$ or e-beam $(\sim 80 \mathrm{kV})$ to the complex until full transformation from inserted fullerene molecules occurred to a helix-like twisted polymer (bent grapheme sheet) inside the CNT (GNR@CNT), or a second CNT inside the CNT (CNT@CNT). Similar to the original approach by Sloan et al. [17] and Smith et al. [10], both encapsulated materials synthesized by Chuvilin et al. [26] had thermodynamic stable energies, given the encapsulating effect from the host-CNT. 
Interestingly, the generated grapheme sheet inside the CNT had a particularly high free-energy $(1.23 \mathrm{eV})$ given its mobile and "dangling" ends inside the CNT. The graphene sheet was therefore stabilized through the addition of sulfur, hydrogen or other heteroatoms, which reduced the gap to the low $0.57 \mathrm{eV}$, inducing a higher chemical and physical stability of the complex. The extensive work by Chuvilin et al. [26] further showed that the sulfur atoms were more stable as atomic ends to the encapsulated carbon chain than oxygen and nitrogen atoms, particularly against e-beam irradiation, given their larger atom weight. The known nature of sulfur atoms in binding to metal clusters, such as gold and platina contacts makes it particularly suitable as a "capping atom" for extending the ends of the encapsulated materials to molecular contacts or conductive metal clusters, with particular application in solar-energy wafer and manufacturing of green-energy components [27]. In this context, sulfur has a weak polarizing atomic character, and does not affect the electronic dipole moment across the encapsulated chain of atoms, as other atoms such as oxygen would do, thereby increasing chemical stability and simultaneously supplying conductivity.

For manufacturing purposes, the addition of sulfur to the encapsulated materials can be performed by adding TTF to the encapsulated molecules $\left(\mathrm{C}_{60}\right.$, or other molecules), or plain TTF inserted directly in the CNT [26].

As for the imaging techniques for encapsulated carbon chains, imaging of growth and assimilation of encapsulated chains of atoms inside CNTs is ideally carried out through the use of time-series of aberration-corrected high resolution transmission electron microscopy (AC-HRTEM) [26]. As mentioned below (vide infra), continuous irradiation from TEM imaging can affect the growth or stability of the chemical components during the intercalation, and therefore require low-energy beams or large intervals during capturing [3]. In the study by Chuvilin et al. [26], the electron irradiation was decreased from 80 to $20 \mathrm{kV}$, giving improved beam monochromaticity.

\section{Encapsulation of metals in CNTs}

The very first study of encapsulation of metal compounds in CNTs was published in 1998 by Sloan and colleagues, where they inserted a $\mathrm{RuCl}_{3}$ solution inside CNTs, for subsequent conversion to elemental ruthenium. The insertion of metal assemblies was also performed by Meyer et al. [28] who inserted potassium halides in CNTs, later followed by insertion of metallofullerenes in CNTs [29], a new approach of inserting metal halides in CNTs [30, 31], and metal-particles and as-prepared metals [32]. La Torre et al. [33] presented in a recent study an approach for a size-specific growth and encapsulation of gold nanoparticles inside graphitized CNF as host-nanocontainers which were used as chemical catalyzers (see Fig. 5). The advantage and strength in advanced manufacturing of this application was the ability to control the size of the nanoparticles during encapsulation

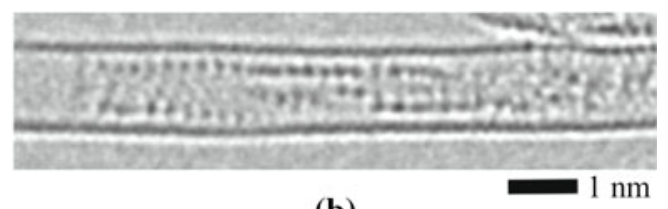

(b)

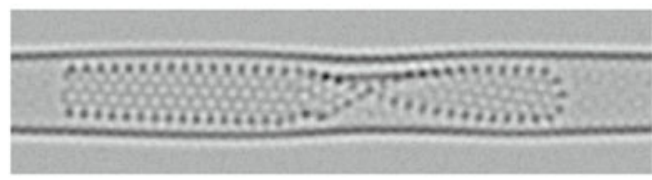

(d)

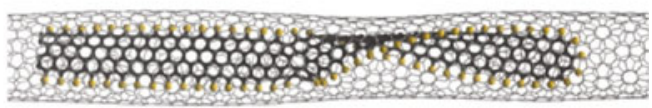

(f)

(e)

Fig. 4 Carbon nanoribbons encapsulated in CNTs through e-beam irradiation of inserted $\mathrm{C}_{60}$. Initial encapsulation of $\mathrm{C}_{60}$ fullerenes makes the foundation for the synthesis of nanoribbons twisted helically inside a CNT with diameters of $10 \AA$ [26] (courtesy of Prof. Andrei N. Khlobystov at the School of Chemistry, University of Nottingham) 
in the hollow tubes, where Ref. [33] reported that the intermolecular interactions between guest nanoparticles and host-nanocontainers play a central role in the modulation of particle size inside CNTs. In addition, the visualization methods, and the process of NP-growth inside the nanocontainers have also a certain effect on the particle size determination during growth. This is caused by the electron beam from the TEM which causes disturbance in the kinetic and energetic processes during growth, which compromises the manufacturing and synthesis of such composites (e.g., Au particles in GNF). TEM can be a useful tool in visualizing the resulting complexes, and discern details at a near-atomic level, however its application in understanding the growth and encapsulation mechanism of particles inside nanocontainers is unsuitable with the present technologies [33].

La Torre et al. [33] applied the use of $n$-pentane and supercritical $\mathrm{CO}_{2}$ at $27.580 \mathrm{MPa}, 40{ }^{\circ} \mathrm{C}$, to obtain the formation of AuNP-GNF (see Fig. 5). The function of supercritical $\mathrm{CO}_{2}$ is exerted through its ultra low viscosity, creating a chemical inert and "slippery" environment for the migration of nanoparticles inside the hollow tubes, delivering nanoparticles inside the tubes without affecting their physical and chemical stability. Additionally, the use of GNF over CNTs included a series of advantages, where for instance the series of steps created in between the conestructures created anchoring points for the nanoparticles (see Fig. 5) [33]. The structural differences between GNF and CNT presents advantages in the case of nanoparticle encapsulation, though lower applicability for more linear chains grown inside, as in the case of the studies by Sloan et al. [34], who encapsulated contiguous linear $\mathrm{AgBr}$ bundles inside CNTs.

Encapsulating metal halides follows a similar protocol as encapsulation of organic molecules [12], where the mixture of metal halides and CNT are prepared and ground before heating in quartz ampoules at $300{ }^{\circ} \mathrm{C}$, and subsequently cooled at room temperature for $3 \mathrm{~h}[30,34]$ (see Fig. 6). Similar studies to the work by Sloan and his group on encapsulating metal complexes inside CNTs include tungsten-sulfide needles [35]. The manufacturing approach for this special combination of conductive metalsulfides with CNT is dependent on a solid-gas reaction, where a small quantity of the metal sulfide $(5 \mathrm{mg}$ ) and the CNTs is heated at $840{ }^{\circ} \mathrm{C}$ with an influx of forming gas $\left(\mathrm{H}_{2} / \mathrm{N}_{2}\right)$. The gas mixture is ideally set to $5 \%$ hydrogen and $95 \%$ nitrogen, given that this particular ratio promotes a uniform effect on the metal-complex morphologies, independently of the flow-ratio of the carrier gases [34, 35]. The advantage of using tungsten particles is in their non-defective surfaces, by tungstens physical properties, enclosing in the nanotubes quite perfectly [35].

The dimensions of the given complexes are in the range of $50 \mathrm{~nm}$ in length and $15 \mathrm{~nm}$ in diameter, and are part dependent on the flow-rate of the forming gas, which

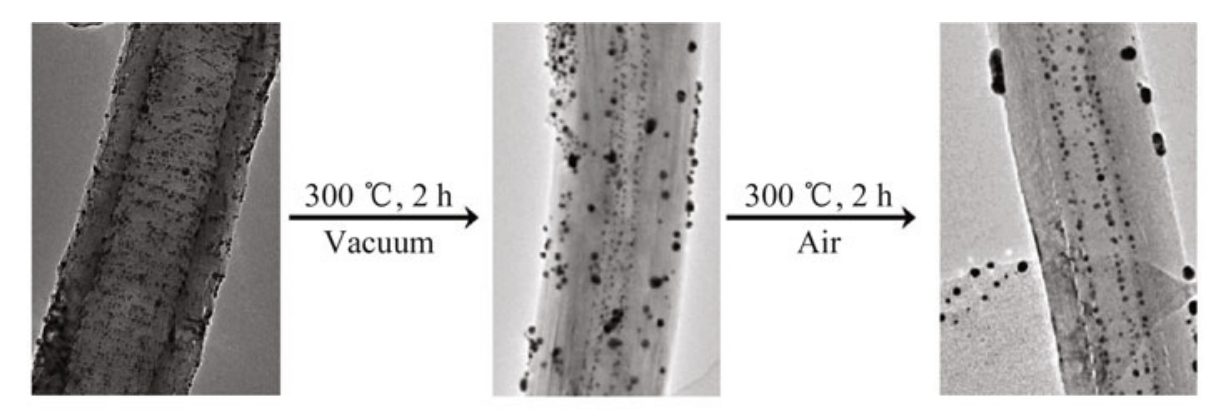

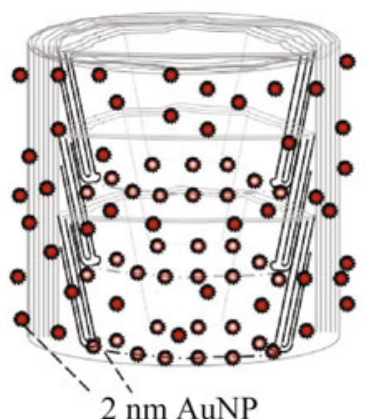

(a)

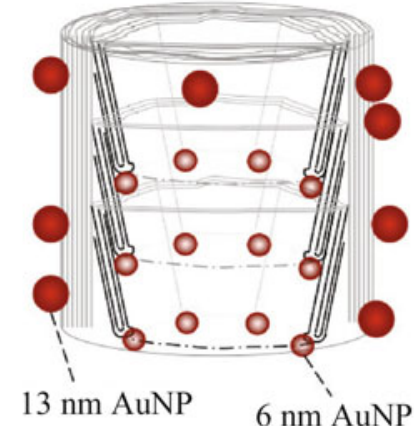

(b)

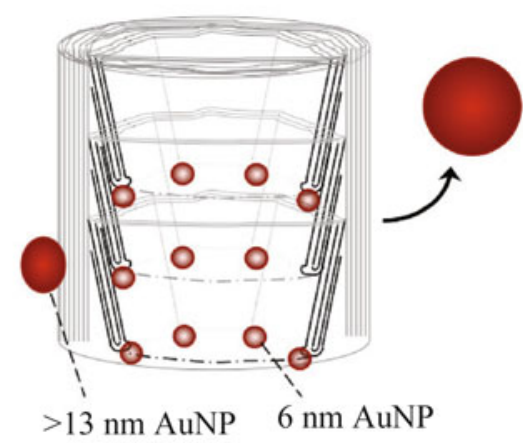

(c)

Fig. 5 Grown gold nanoparticles inside graphene nanotubes (GNT) [33] a $2 \mathrm{~nm}$ particle size, encapsulated in GNT, b after $2 \mathrm{~h}$ heating at $300{ }^{\circ} \mathrm{C}$, particle size grown to $13 \mathrm{~nm}$, c particle grown above $13 \mathrm{~nm}$ in diameter after $4 \mathrm{~h}$ at $300^{\circ}$ from start (courtesy of Dr. Maria Del Carmen Gimenez-Lopez at the School of Chemistry, University of Nottingham) 


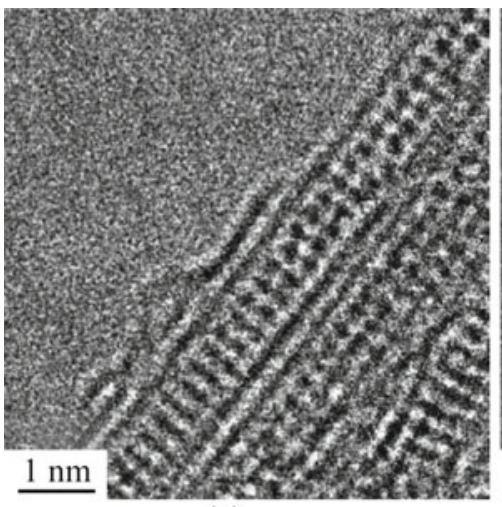

(a)

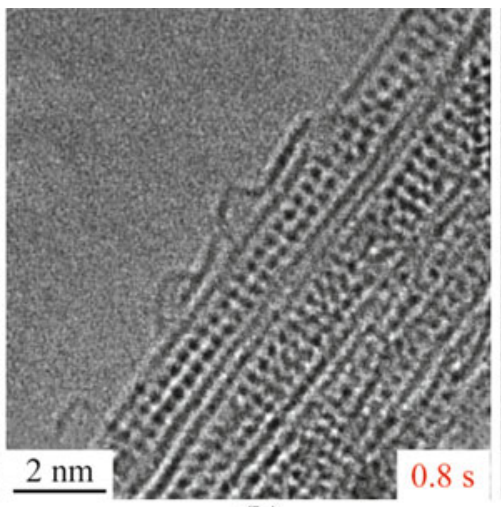

(b)

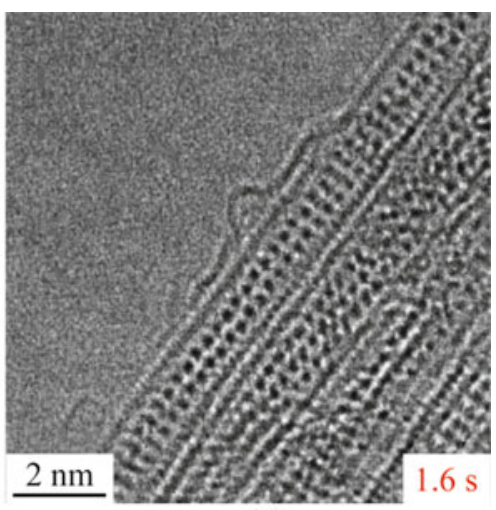

(c)

Fig. 6 Time-lapse HRTEM images of encapsulated KI crystals in CNTs (previously unpublished) (courtesy of Dr. Jeremy Sloan, University of Warwick, Warwick Centre of Analytical Science). The KI crystals were trapped inside tubular CNT structures as reported in Refs. [30, 33]

affects the shape forming of the particles when the ratio of gases exceeds a certain limit, either tungsten or nanotubes particles appear without any merging effects.

$\mathrm{Xu}$ et al. [36] inserted lanthanide-halide, neodyniumhalide, terbium-halide, samarium-halide, gadolinium- and europium-halide crystals inside nanotubes at $20 \%-40 \%$ yield ratio, under similar conditions as in the tungstensulfide study by Rothschild et al. [35]. Similar to the methods by Sloan et al. [34] on metal-halide encapsulation in CNTs, the anhydrous high-energy metal halides were ground and mixed with CNTs under dry-box conditions, and heated up to melting temperatures of the respective metal-halides $\left(609-860{ }^{\circ} \mathrm{C}\right)$. The inserted crystals form zig-zag arrangements of halide polyhedrals (octagonal and hexagonal architectures), which appear clearly under HRTEM [38]. The most impressive achievement in metal encapsulation in CNTs was published by Del Carmen Gimenez-Lopez et al. [37] who inserted $\mathrm{Mn}_{12} \mathrm{Ac}$ single molecular magnets in CNTs. Their manufacturing and methodological approach included the use of supercritical $\mathrm{CO}_{2}$ as in the study by La Torre et al. [33], which exerts low viscosity, high diffusivity and zero surface tension, and facilitates an easy and unrestricted insertion of the molecular magnets inside the CNT. The complexes produced by Del Carmen Gimenez-Lopez et al. [37] yielded modulated electromagnetic properties of the CNTs, by the strong magnetic forces of the inserted magnets. This study shows that several advantages arise when encapsulating metal clusters and atoms inside nanotubes, particularly in that the shaping form of the CNT results in a diametercontrolled polymorphism of encapsulated inorganic materials, as mentioned by Guan et al. [38], which also promotes an increased anisotropy of the tubular structures [39].

In further context with electromagnetic and energetic metal-based molecules, Del Carmen Gimenez-Lopez et al. [4] inserted $\mathrm{Mn}_{3} \mathrm{O}_{4}$ nanobricks into herringbone-formed
CNF and GNF, after a suspension of metal oxide particles in hexane, diluted in supercritical $\mathrm{CO}_{2}$ (at $27.580 \mathrm{MPa}$, $40{ }^{\circ} \mathrm{C}$ ). Interestingly, the packing of the metal oxide particles gave fully aligned particles in the GNF, while random orientations of the metal oxide particles in the CNF (see Fig. 7), which can affect the conductive properties of these two types of oxide-composites (graphene over carbon fibers). However, a considerable advantage of the assimilation of packed metal oxides in the CNFs was their higher packing density ( $26 \%$ vs. $11 \%$ ) than in GNFs. The study by Del Carmen Gimenez-Lopez et al. [4] showed that the most magnetic material was the CNF composite, given its higher packing density of metaloxide (see Fig. 7). The study by Del Carmen Gimenez-Lopez et al. [4] also showed that larger nanobricks were encapsulated when CNFs were used, over GNF (see Fig. 7). Reference [4] showed that the combination of magnetic materials and CNTs (or GNF, CNF) is a promising material for modulation of physical properties in advanced materials, and based on the manufacturing method, presents a highly reproducible method for a larger scale approach. Other methods for this combinatory approach in manufacturing of exotic combinations of metals and nanotubes are capillarity filling of the molten metal salts, with applied pyrolysis of the encapsulated material [40]. However these methods do not have sufficient control over the composition, size and morphology of the nanoparticles formed inside the tubular structures. The methods of encapsulation with most precise approach, making the most detailed maneuvers possible, represent the state-ofthe-art approaches for manufacturing magnetic and highenergy metal-CNT complexes [41]. The applicability of such novel materials and their manufacturing methods holds promising potentials for the nanoengineering of spintronic devices, optoelectronic components, and junctions and molecular contacts. 


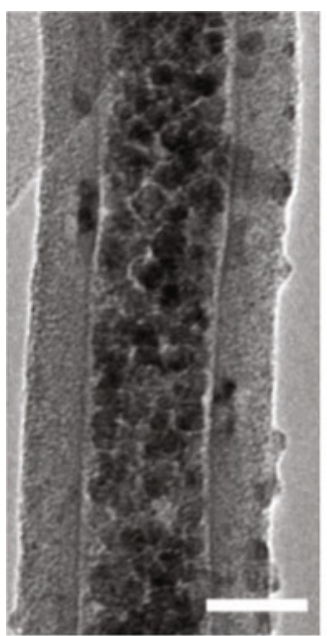

(a)

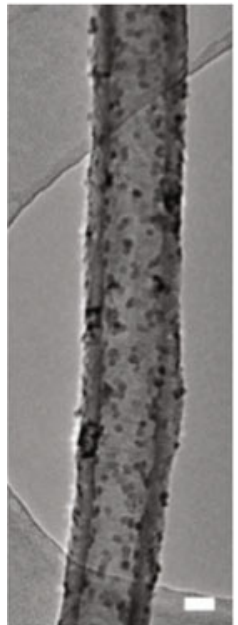

(e)

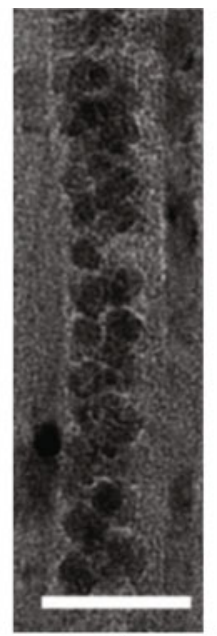

(b)

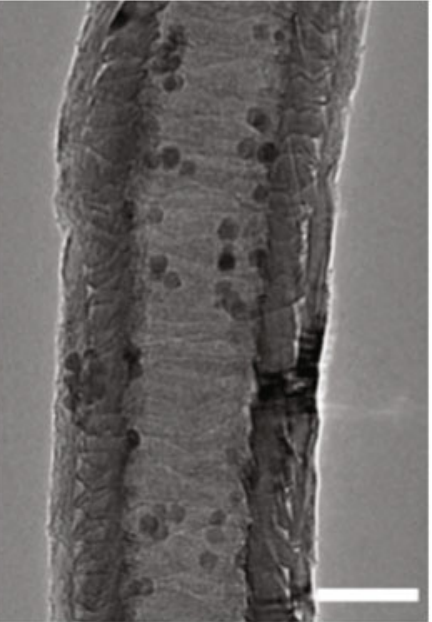

(f)

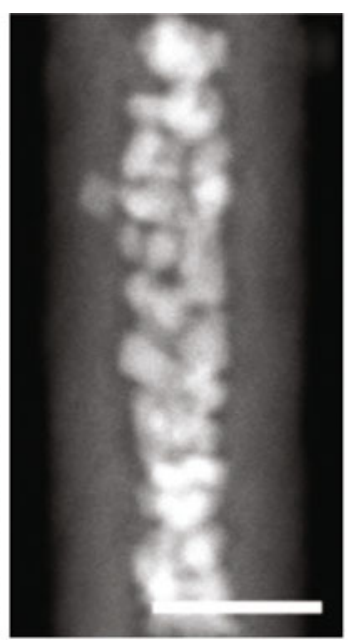

(c)

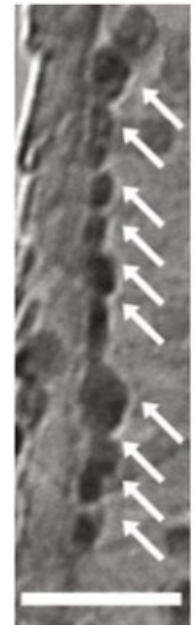

(g)

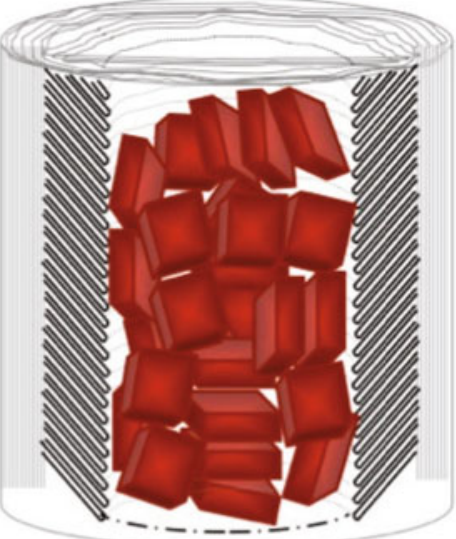

(d)

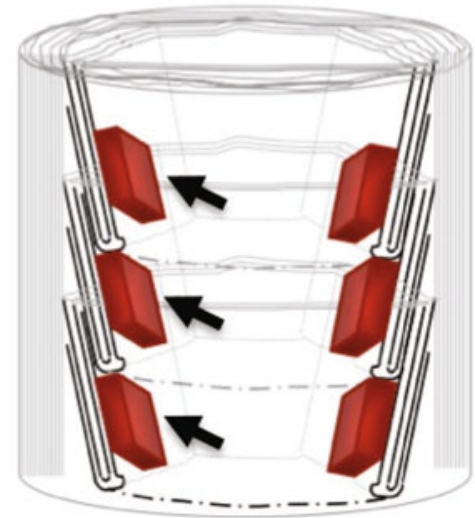

(h)

Fig. 7 Encapsulated $\mathrm{Mn}_{3} \mathrm{O}_{4}$ nanoparticles in GNFs a-c TEM images of $\mathrm{Mn}_{3} \mathrm{O}_{4}$ nanoparticles in $\mathrm{CNF}$; d model illustration of $\mathrm{Mn}_{3} \mathrm{O}_{4}$ nanoparticles encapsulated in CNFs; e-g bright-field TEM images of $\mathrm{Mn}_{3} \mathrm{O}_{4}$ adsorbed in the step edges of GNFs; $\mathbf{h} \mathrm{Mn}_{3} \mathrm{O}_{4}$ nanoparticle encapsulated in GNFs (courtesy of Dr. Maria Del Carmen Gimenez-Lopez at the School of Chemistry, University of Nottingham)

\section{Encapsulation of polymers in CNTs}

Encapsulation of polymers in CNT is perhaps the least explored avenue in encapsulation methods, encompassing only three known studies of successful growth of polymers inside CNTs [42-44].

All three studies have endeavored the encapsulation of carbon-based polymers, respectively polystyrene, polyacetylene and polyethyleoxide for the purpose of enhancing the structural properties of CNTs. In Ref. [42], the encapsulation was performed inside MWCNTs, with an outer diameter of $40-50 \mathrm{~nm}$ and a length of $3 \mu \mathrm{m}$ which were purified to $>95 \%$. The CNT were mixed at a ratio of $20 \mathrm{mg}$ with $2 \mathrm{~mL}$ styrene, along with polymerization agent (benzoyl peroxide). The reagents were accommodated in a glass vial into a stainless steel vessel, applied to ultrasonication for dispersing CNT fully in the monomer solution of styrene. Supercritical $\mathrm{CO}_{2}$ was then introduced at a pressure of $12 \mathrm{MPa}$, and the solution was left for soaking for $2 \mathrm{~h}$. Subsequently, the $\mathrm{CO}_{2}$ was released from the carrier, and the vial was transferred to an oven at $100{ }^{\circ} \mathrm{C}$ for initiating the polymerization reaction [42]. The reaction was allowed to proceed for $24 \mathrm{~h}$, and then stopped by cooling to room temperature. Necessary washing of the inactivated reactants and side-products was performed with pure toluene, and subsequently filtered and vacuum dried. The procedure by Liu et al. [42] is a potential template protocol for other polymerization methods and is shown in Fig. 8.

In the second approach for polymerization, Steinmetz et al. [43] applied polyacetylene as a filling material in MWCNTs. The CNTs were refluxed with $\mathrm{HNO}_{3}$ for $8 \mathrm{~h}$, and then filtered on a membrane. The CNTs were then 


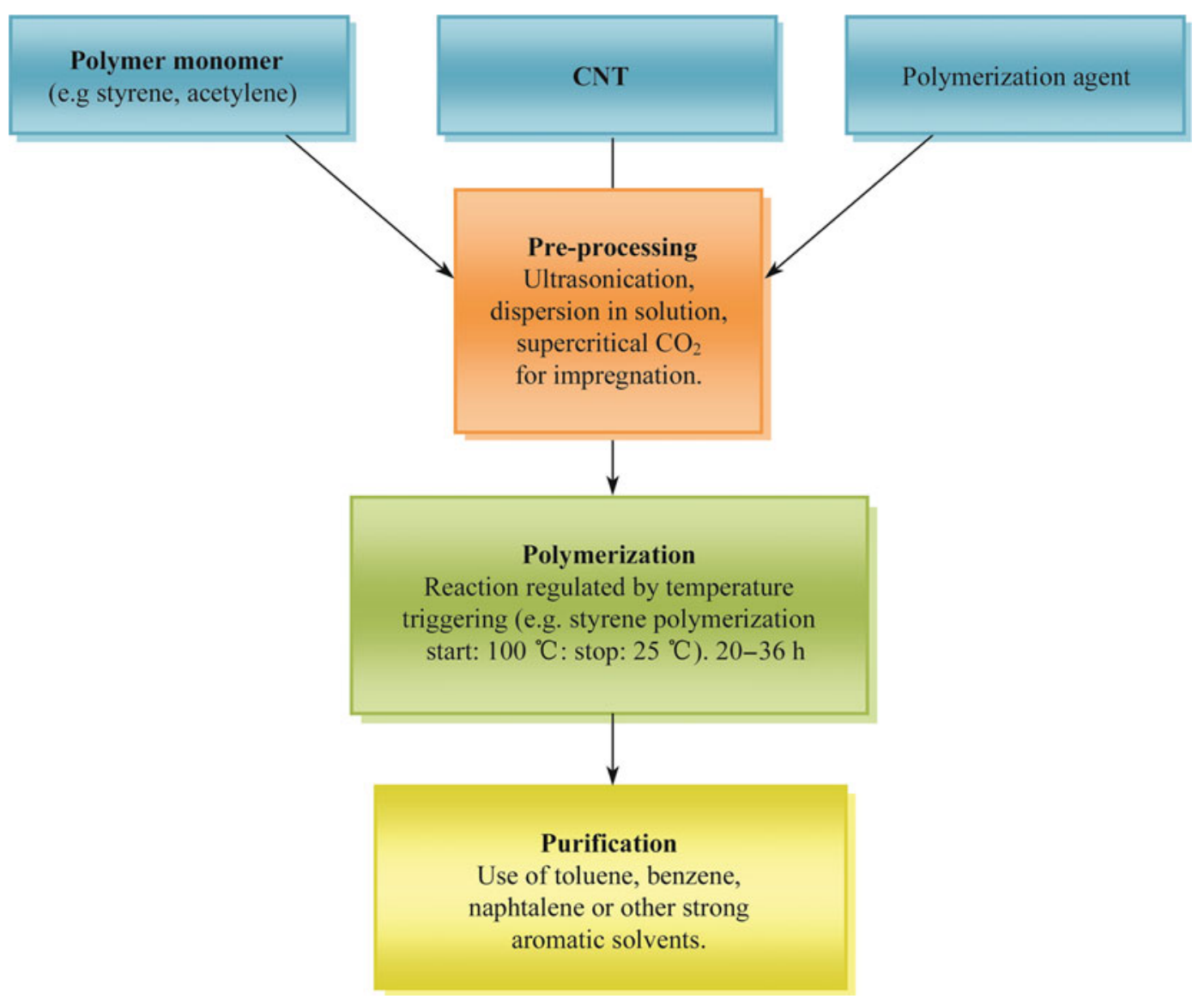

Fig. 8 Main steps for polymer growth inside CNTs with polystyrene [42]

washed with a strong base $(\mathrm{KOH})$ for the thorough removal of small oxidized particles. The CNTs were then ground with a mortar, and filled with toluene using supercritical $\mathrm{CO}_{2}$ in a high pressure vessel equipped with a pressure gauge, thermometer and a magnetic stirrer. The subsequent reactions were carried out using a Ziegler-Natta catalyst, under argon atmosphere. The toluene-filled tubes were stirred overnight in the catalyst, and a solution of triethylaluminium and tetrabutyltitanate were added in toluene. The nanotubes were then washed several times, and dried for removing the catalyst outside the tubular structures. The dried tubes were then transferred to a flask filled with acetylene gas at $0.5 \mathrm{kPa}$ and let to react overnight. A control sample was also prepared without removal of the catalyst, and both showed apparent growth of polyacetylene inside the CNT. The use of catalyst led to some impurities in the composite, visible in the electron microscope. However the polymerized acetylene was only confirmed using ${ }^{1} \mathrm{H}$ NMR technique, given that it was not visible with TEM imaging.

Bazilevsky et al. [44] published a method for filling asgrown $100 \mathrm{~nm}$ diameter MWCNTs at room temperature with low-molecular weight polyethyleneoxide and polycaprolactone polymers, by the spontaneous intercalation through the open ends of the MWCNTs. In their study, low-molecular weight polymers filled the graphitic nanotubes at higher yields than heavier polymers, albeit the heavy polymers still intercalated without full protrusion through the CNT cavity. The procedure was carried out by immersing the nanotubes in a dilute polymer solution, after being purified in ionized water through sonication. The immersed CNTs in diluted polymer solutions resulted as $100 \mathrm{~nm}$ thick films, suitable for TEM imaging [44].

An additional feat on polymerization inside CNTs was published in 2005, by Britz et al. [45] who polymerized a series of fullerenes inside a CNT. The procedure encompassed an insertion of fullerene epoxide molecules inside CNTs at $50{ }^{\circ} \mathrm{C}$ using supercritical $\mathrm{CO}_{2}$ over a period of 6 day. With a yield of $70 \%$, the group heated the complex to $260{ }^{\circ} \mathrm{C}$ for 3 day, which triggered the polymerization of the fullerene epoxides to form $\left(\mathrm{C}_{60}-\mathrm{O}\right)_{n}$ chains inside the nanotubes. As also performed by Liu et al. [42], the initial insertion of the monomer for a polymerization reaction is followed by a temperature triggering after successful encapsulation, which 
does not affect the CNT, and starts the polymerization inside the CNT by merely temperature change.

Currently, only these four studies have been reported on the intercalation of polymers inside CNTs, which represent an interesting avenue of research for novel materials for applications in nanoelectronics and microengineering. In particular, polymerization of molecules inside CNTs presents an opportunity for the automatization in manufacturing of such composites, applicable to several routes of research. For instance, polymerization of acetylene in a CNT yields composites with conductive properties, with higher conductivity than many other carbon-based conducting molecules/polymers [46-49]. For further developments of polymerization reactions in CNTs, other options to be considered for manufacturing approaches involve a variety of hydrogels such as polyols, which bear polar and hydrophilic properties $[50,51]$ and can also be polymerized inside CNTs, using temperature-based reaction-triggering. The inclusion of such alternatives may increase the applicability of CNTs in other areas of interest, such as sensing, electron transfer/ transport and catalysis. Other relevant works to this rationale are published by Oh et al. [52] and Metyjaszewski et al. [53] who reported the preparation of nanostructured hybrid hydrogels with polar groups and a variety of conductive

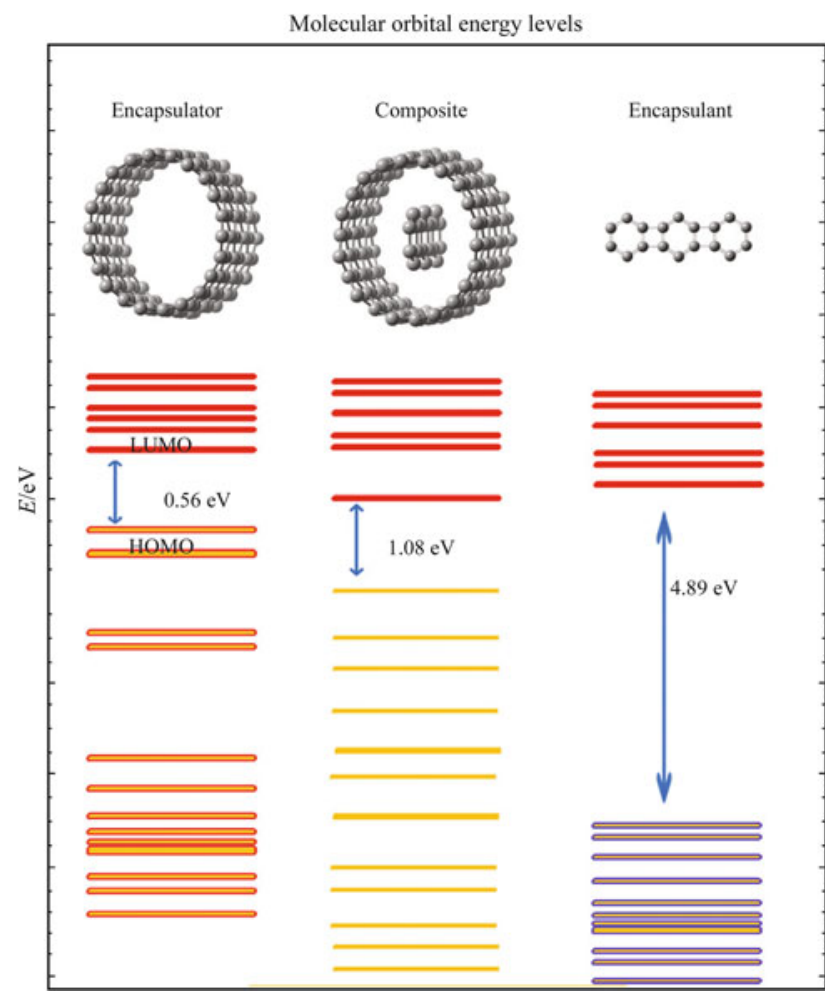

Fig. 9 Energy levels and gap relationships between encapsulator (CNT) and encapsulant (triphenylene) carbon-groups. Linear and conductive chains can in other words be grown inside the CNT as a novel manufacturing method, suitable for advances in manufacturing.

\section{Effects from encapsulation}

Encapsulation of molecules, crystals and other ensembles of atoms inside CNTs can promote a new field of nanosciences as reported in Ref. [54], where the chemical properties of molecules in enclosed chambers carry out designated functions, such as catalysis, conductance and potential regulation of reactions rates, such as clustering reactions of crystals and ionic polyhedral [54]. The large surface areas inside the tubular structures of the CNTs functions as a surface with potentially catalytic and chemically stabilizing effects, and the application of e-beam can be used to affect and trigger chemical processes such as coalescence of carbon-particles, polymerization and other steps towards altered physical qualities for manufacturing and fabrication $[54,55]$. The effects from encapsulating materials are primarily of three classes: modulated thermal and electrical conductance of the CNT, altered chemical stability and increased physical

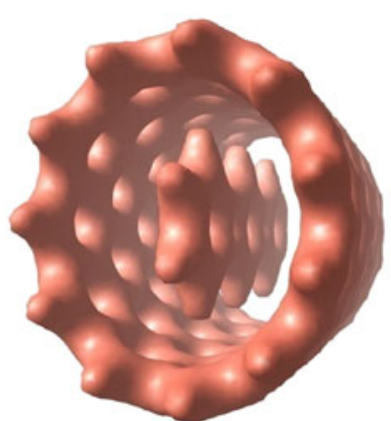

(a)

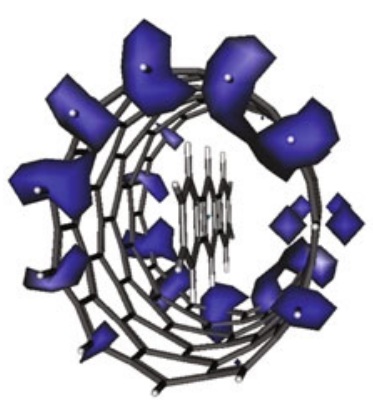

(c)

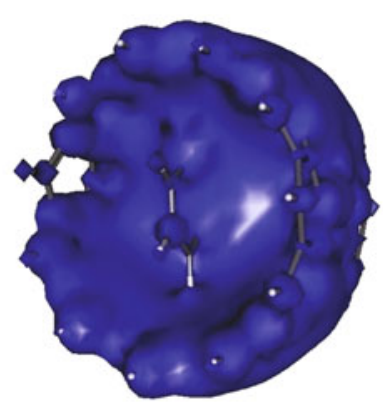

(b)

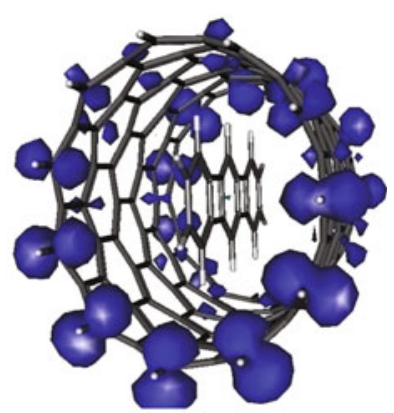

(d)
Fig. 10 Electron density of the encapsulated molecule triphenylene encapsulated inside a CNT a Isosurface of the electron density; b electrostatic potential map depicting the electrostatic forces at play in the composite; c Fukui-surface of electrophilicity, which indicates a potential route of transport during $n$-type operation (electrontransport); d Fukui-surface of nucleophilicity, which indicates a potential route of transport during $p$-type operation (hole-transport) 
robustness. The changes occurring at the physical level can be partly explained from the electronic level, via theoretical studies of structures and ensembles of encapsulated molecules and chains inside CNTs. In an electronic study, Ref. [8] showed that the changes in conductive properties of CNTs after insertion a molecule caused increased chemical stability of the ensemble, resulting in a prolonged chemical half-life and the material strength. The increased stability is caused by the electrostatic tension between the inserted molecule and the intra-tubular face of the CNT, effectively raising the oxidation potential of the entire system [8]. Such an effect was also reported by Dinadayalane et al. [9] who showed that the interaction energy increases with the length of the linear carbon chain encapsulated in the CNT, inducing a dimension-dependent electronic effect. Other studies [56-62] have showed that molecules can enter spontaneously given the potential energy-vacuum in the CNTs, making the encapsulation energetically favorable, and that the interaction energy also known as compression energy (arising between the molecule and the nanotubes) creates a high electrostatic tension which can affect conductance and physical properties of the composite. Encapsulation promotes also increased stability of the $\pi$-electrons on the surface of the nanotubes from oxidative attacks changing their ability to acts as either $n$ - and $p$-type conductors during operation $[12,57]$.
For a direct illustration of the effects of encapsulation, a system with a triphenylene molecule encapsulated in a CNT was studied using a density functional theory approach with the BH and HLYP method and 3-21G basis set [63]. The individual (out of complex) HOMO-LUMO gap of triphenylene resulted at $4.89 \mathrm{eV}$, while the CNT (1 nm diameter, $1.5 \mathrm{~nm}$ length) has a gap of $0.56 \mathrm{eV}$. The composite has an HOMO-LUMO gap of $1.08 \mathrm{eV}$. Interestingly, the encapsulation resulted in a significant change in electronic gap properties (see Fig. 9), where the electrostatic forces are shown to exert their effect on the encapsulated system, forcing the electrons closer to one another, and creating an intra-tubular tension field (see Fig. 10). In addition, an interesting feature pointed by Dr. $\mathrm{Lu}$ (personal communication) of the encapsulation of the fully-carbon based triphenylene molecule in a CNT, is that the gaps of each respective component (nanocontainerCNT and guest molecule-triphenylene) are not close to one another (see Fig. 9), creating two different gap levels, which yield a Fukui frontier separation between the two molecules (see Fig. 10d). Figure 9 illustrates the differences in gap widths for the three different systems analyzed for conductive properties using the DFT method BHandHLYP with the 3-21G basis set [63]. Energy levels are illustrative for graphical simplicity, however the gapmagnitudes between the three states are relative to

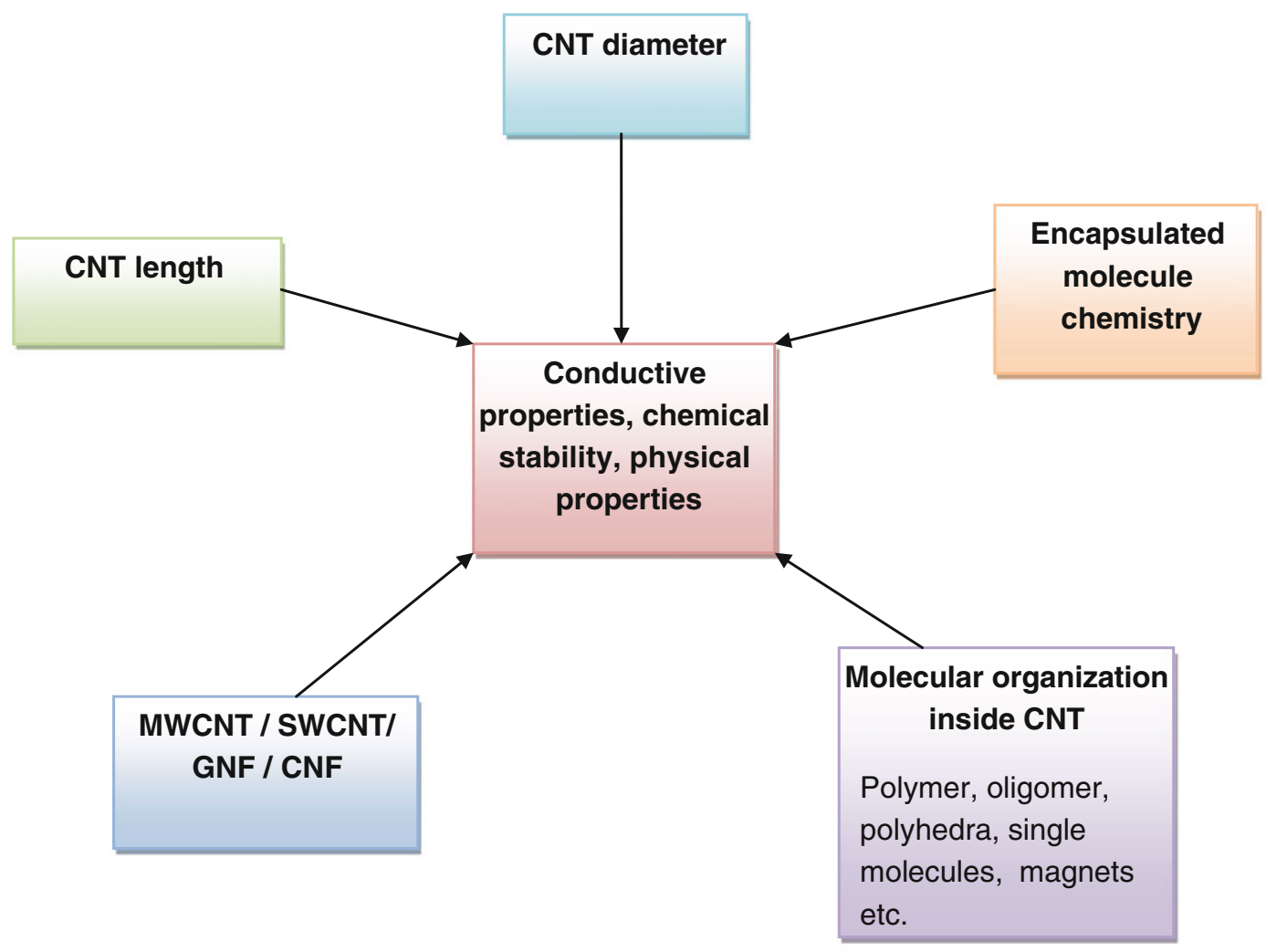

Fig. 11 Central factors affecting the chemical, electronic and physical qualities of manufactured encapsulated materials 
calculations. This Fukui separation generates a conductance separation between the molecules from the quantum level. This particular phenomenon creates two conductance levels in one system, presenting opportunities for quantum conductance and nanoelectronic circuits, for the development of separated conductive units with gap-barriers.

Inversely, when the gap levels of the two entities are adjusted closer to one another through the change of encapsulated molecule or slight molecular modifications of the existing guest, the conductance of the two parts-the CNT and the encapsulated molecule can be tuned to one another, giving unique conductive properties of the composite.

Finally, the diameter of the CNT plays a major role in the modulation of the total gap when filled with guest species. In the current model analyzed, a CNT-diameter of $9 \AA$ yields an approximate HOMO-LUMO gap with the given DFT method of calculation results at $1.08 \mathrm{eV}$. When the diameter is increased to $10 \AA$, the approximate gap is reduced to $0.816 \mathrm{eV}$, and furthermore reduced to $0.672 \mathrm{eV}$ when diameter of the CNT is increased to $11 \AA$. Although dependent on the molecular identity and chemistry of the guest molecule, the modulation of gap by diameter change in a composite plays a major role for a nanoelectronic scenario, introducing the crucial relationship of the spatial vicinity between encapsulant orbitals and CNT orbitals, demanding further analysis.

Nevertheless, by modulating the dimensions of the CNT, the atomic content and bonding-nature of the inserted molecule, the identity of the inserted molecule and other chemical properties of the composite, result in valuable changes in the properties of the system in terms of conductance, chemical stability, thermal conductance and other factors (see Fig. 11).

\section{Conclusions}

A review of the current and previous methods for encapsulating a variety of molecules, including molecular chains, polymers and metal complexes has been presented. The reviewed work indicates a promising development for the incorporation of advanced materials in the available landscape of manufactured circuits, nanounits and connecting networks in consumer- and nano-electronics. A comprehensive consideration of central factors for the modulation of electronic and chemical properties of encapsulated materials has also been briefly introduced, directing the future work of advanced manufacturing of composites and encapsulated materials towards a further development of methods and approaches to be applied in novel technologies. Additional sources and reviews on the topic presented herein are suggested as further reading [25, 54].
Acknowledgments The author would like to thank Dr. Maria del Carmen Gimenez-Lopez and Prof. Andrei N. Khlobystov from the University of Nottingham for the images included herein and helpful comments on the review. The author would also like to thank Prof. Jeremy Sloan from the University of Warwick for also supplying previously non-published images of the KI crystals, images of fullerenes in CNTs and useful comments. Grateful acknowledgements go also to Dr. Tian Lu from the University of Beijing for useful comments on the separated gap energies in the composite analysis. Finally, the author would like to thank Prof. van der Spoel, at the Computational Systems and Biology Group at Uppsala for the guest-researcher position.

\section{References}

1. Guan L, Shi Z, Li M et al (2005) Ferrocene-filled single-walled carbon nanotubes. Carbon 43:2780-2785

2. Obergfell D, Meyer JC, Haluska M et al (2006) Transport and TEM on dysprosium metallofullerene peapods. Phys Status Solidif B 243:3430-3434

3. Del Carmen Gimenez-Lopez M, Chuvilin A, Kaiser U et al (2011) Functionalised endohedral fullerenes in single-walled carbon nanotubes. Chem Commun (Camb) 47:2116-2118

4. Del Carmen Gimenez-Lopez M, La Torre A, Fay MW et al (2013) Assembly and magnetic bistability of $\mathrm{Mn}_{3} \mathrm{O}_{4}$ nanoparticles encapsulated in hollow carbon nanofibers. Angew Chem Int Ed Engl 52:2051-2054

5. Faist J, Capasso F, Sirtori C et al (1995) Continuous wave operation of a vertical transition quantum cascade laser above $T=80$ K. Appl Phys Lett 67:3057-3059

6. Tóth G, Lent CS (2001) Quantum computing with quantum-dot cellular automata. Phys Rev A 63:052315

7. Lindner NH, Refael G, Galitski V (2011) Floquet topological insulator in semiconductor quantum wells. Nat Phys 7:490-495

8. Meunier V, Sumpter BG (2005) Amphoteric doping of carbon nanotubes by encapsulation of organic molecules: electronic properties and quantum conductance. J Chem Phys 123:24705

9. Dinadayalane TC, Gorb L, Simeon T et al (2007) Cumulativeinteraction triggers unusually high stabilization of linear hydrocarbon inside the single-walled carbon nanotube. Int J Quantum Chem 107:2204-2210

10. Smith BW, Monthoux M, Luzzi DE (1998) Encapsulated C60 in carbon nanotubes. Nature 396:323-324

11. Maniwa Y, Kumazawa Y, Saito Y et al (1999) Anomaly of X-ray diffraction profile in single-walled carbon nanotubes. Jpn J Appl Phys Lett 38:L668-L670

12. Takenobu T, Takano T, Shiraishi M et al (2003) Stable and controlled amphoteric doping by encapsulation of organic molecules inside carbon nanotubes. Nat Mater 2:683-688

13. Morgan DA, Sloan J, Green ML (2002) Direct imaging of o-carborane molecules within single walled carbon nanotubes. Chem Commun 20:2442-2443

14. Smith BW, Luzzi DE (2000) Formation mechanism of fullerene peapods and coaxial tubes: a path to large scale synthesis. Chem Phys Lett 321:169-174

15. Kiang CH, Choi JS, Tran TT et al (1999) Molecular nanowires of $1 \mathrm{~nm}$ diameter from capillary filling of single-walled carbon nanotubes. J Phys Chem B 103:7449-7451

16. Sloan J, Hammer J, Zwiefka-Sibley M et al (1998) The opening and filling of single walled carbon nanotubes (SWTs). Chem Commun 3:347-348

17. Sloan J, Dunin-Borkowski RE, Hutchison JL et al (2000) The size distribution, imaging and obstructing properties of C60 and higher fullerenes formed within arc-grown single walled carbon nanotubes. Chem Phys Lett 316:191-198 
18. Zhang Y, Iijima S, Shi Z et al (1999) Defects in arc-dischargeproduced single-walled carbon nanotubes. Philos Mag Lett 79:473-479

19. Wang ZX, Ke XZ, Zhu ZY et al (2000) Carbon-atom chain formation in the core of nanotubes. Phys Rev B 61:R2472-R2474

20. Warner J, Rümmeli MH, Bachmatiuk A et al (2010) Structural transformations of carbon chains inside nanotubes. Phys Rev B 81:155419

21. Nishide D, Dohi H, Wakabayashi $\mathrm{T}$ et al (2006) Single-wall carbon nanotubes encaging linear chain $\mathrm{C}_{10} \mathrm{H}_{2}$ polyyne molecules inside. Chem Phys Lett 428:356-360

22. Zhao X, Ando Y, Liu Y et al (2003) Carbon nanowire made of a long linear carbon chain inserted inside a multiwalled carbon nanotube. Phys Rev Lett 90:187401-187404

23. Sheng L, Jin A, Yu L et al (2012) A simple and universal method for fabricating linear carbon chains in multiwalled carbon nanotubes. Mater Lett 81:222-224

24. Koshino M, Tanaka T, Solin N et al (2007) Imaging of single organic molecules in motion. Science 316:853

25. Chamberlain TW, Biskupek J, Rance GA et al (2012) Size, structure, and helical twist of graphene nanoribbons controlled by confinement in carbon nanotubes. ACS Nano 6:3943-3953

26. Chuvilin A, Bichoutskaia E, Gimenez-Lopez MC et al (2011) Self-assembly of a sulfur-terminated graphene nanoribbon within a single-walled carbon nanotube. Nat Mater 10:687-692

27. Tang J, Huo Z, Brittman S et al (2011) Solution-processed coreshell nanowires for efficient photovoltaic cells. Nat Nanotechnol 6:568-572

28. Meyer RR, Sloan J, Dunin-Borkowski RE et al (2000) Discrete atom imaging of one-dimensional crystals formed within singlewalled carbon nanotubes. Science 289:1324-1327

29. Lee J, Kim H, Kahng SJ et al (2002) Bandgap modulation of carbon nanotubes by encapsulated metallofullerenes. Nature 415 : 1005-1008

30. Sloan J, Kirkland AI, Hutchison JL et al (2002) Integral atomic layer architectures of $1 \mathrm{D}$ crystals inserted into single walled carbon nanotubes. Chem Commun: 1319-1332. doi:10.1039/B200537A

31. Bendall JS, Ilie A, Welland ME et al (2006) Thermal stability and reactivity of metal halide filled single-walled carbon nanotubes. J Phys Chem B 110:6569-6573

32. Zhou J, Song H, Chen X et al (2010) Diffusion of metal in a confined nanospace of carbon nanotubes induced by air oxidation. J Am Chem Soc 132:11402-11405

33. La Torre A, Del Carmen Gimenez-Lopez M, Fay MW et al (2012) Assembly, growth, and catalytic activity of gold nanoparticles in hollow carbon nanofibers. ACS Nano 6:2000-2007

34. Sloan JM, Wright D, Bailey S et al (1999) Capillarity and silver nanowire formation observed in single walled carbon nanotubes. Chem Commun: 699-700. doi:10.1039/A901572H

35. Rothschild A, Sloan J, Tenne R (2000) Growth of WS2 nanotubes phases. J Am Chem Soc 122:5169-5179

36. Xu C, Sloan J, Brown G et al (2000) 1D lanthanide halide crystals inserted into single-walled carbon nanotubes. Chem Commun 24:2427-2428

37. Del Carmen Gimenez-Lopez M, Moro F, La Torre A et al (2011) Encapsulation of single-molecule magnets in carbon nanotubes. Nat Commun 2:407

38. Guan L, Suenaga K, Shi Z et al (2007) Polymorphic structures of iodine and their phase transition in confined nanospace. Nano Lett 7:1532-1535

39. Philip E, Sloan J, Kirkland AI et al (2003) An encapsulated helical one-dimensional cobalt iodide nanostructure. Nat Mater 2:788-791

40. Ugarte D, Chatelain A, de Heer WA (1996) Nanocapillarity and chemistry in carbon. Science 274:1897-1899
41. Gubin SP, Koksharov YA (2002) Preparation, structure, and properties of magnetic materials based on co-containing nanoparticles. Inorg Mater 38:1085-1099

42. Liu Z, Dai X, Xu J et al (2004) Encapsulation of polystyrene within carbon nanotubes with the aid of supercritical $\mathrm{CO}_{2}$. Carbon $42: 458-460$

43. Steinmetz J, Kwon S, Lee HJ et al (2006) Polymerization of conducting polymers inside carbon nanotubes. Chem Phys Lett 431:139-144

44. Bazilevsky AV, Sun K, Yarin AL et al (2007) Selective intercalation of polymers in carbon nanotubes. Langmuir 23: 7451-7455

45. Britz DA, Khlobystov AN, Porfyrakis K et al (2005) Chemical reactions inside single-walled carbon nano test-tubes. Chem Commun 107:37-39

46. Ito T, Shirakawa H, Ikeda S (1975) Thermal cis-trans isomerization and decomposition of polyacetylene. J Polym Sci 12: 1943-1950

47. Chiang CK, Fincher CB, Park YW et al (1977) Electrical conductivity in doped polyacetylene. Phys Rev Lett 39:1098-1101

48. Chiang CK, Druy MA, Gau SC et al (1978) Synthesis of highly conducting films of derivatives of polyacetylene, $(\mathrm{CH})_{x}$. J Am Chem Soc 100:1013-1015

49. Shirakawa H, Louis EJ, MacDiarmid AG et al (1977) Synthesis of electrically conducting organic polymers: halogen derivatives of polyacetylene, $(\mathrm{CH})_{x}$. J Am Chem Soc: 578

50. Ravve A (2012) Principles of polymer chemistry. Springer, New York

51. McCormick CL, Kirkland SE, York AW (2006) Synthetic routes to stimuli-responsive micelles, vesicles, and surfaces via controlled/ living radical polymerization. J Macromol Sci C 46:421-443

52. Oh JK, Drumright R, Siegwart DJ et al (2008) The development of microgels/nanogels for drug delivery applications. Prog Polym Sci 33:448-477

53. Matyjaszewski K, Tsarevsky NV (2009) Nanostructured functional materials prepared by atom transfer radical polymerization. Nat Chem 1:276-288

54. Khlobystov AN (2011) Carbon nanotubes: from nano test tube to nano-reactor. ACS Nano 5:9306-9312

55. Chamberlain TW, Gimenez-Lopez MdC, Khlobystov AN (2010) Carbon nanotubes as containers. In: Carbon nanotubes and related structures. Wiley-VCH Verlag GmbH \& Co. KGaA, Weinheim, pp 349-384

56. Shi XQ, Dai ZX, Zhong GH et al (2007) Spin-polarized transport in carbon nanowires inside semiconducting carbon nanotubes. J Phys Chem C 111:10130-10134

57. Tran-Duc T, Thamwattana N (2011) Modeling encapsulation of acetylene molecules into carbon nanotubes. J Phys 23:225302

58. Lee SU, Belosludov RV, Mizuseki H et al (2011) Electron transport characteristics of organic molecule encapsulated carbon nanotubes. Nanoscale 3:1773-1779

59. Ilie A, Bendall JS, Nagaoka K et al (2011) Encapsulated inorganic nanostructures: a route to sizable modulated, noncovalent, on-tube potentials in carbon nanotubes. ACS Nano 5:2559-2569

60. Kuwahara R, Kudo Y, Morisato T et al (2011) Encapsulation of carbon chain molecules in single-walled carbon nanotubes. J Phys Chem A 115:5147-5156

61. McIntosh GC, Tomanek D, Park YW (2003) Energetics and electronic structure of a polyacetylene chain contained in a carbon nanotube. Phys Rev B 67:125419

62. Kim G, Kim Y, Ihm J (2005) Encapsulation and polymerization of acetylene molecules inside a carbon nanotube. Chem Phys Lett 415:279

63. Becke A (1998) Density-functional exchange-energy approximation with correct asymptotic behavior. Phys Rev A 38:3098-3100 